\title{
Quantitative Measurement of CSF in Patients with Spontaneous Intracranial Hypotension
}

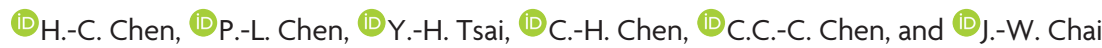

\begin{abstract}
BACKGROUND AND PURPOSE: CSF hypovolemia is a core feature of spontaneous intracranial hypotension. Spontaneous intracranial hypotension is characterized by orthostatic headache and radiologic manifestations, including CSF along the neural sleeves, diffuse pachymeningeal enhancement, and/or venous engorgement. However, these characteristics are only qualitative. Quantifying intraspinal CSF volumes could improve spontaneous intracranial hypotension diagnosis and evaluation of hypovolemic statuses in patients with spontaneous intracranial hypotension. The purpose of this study was to compare intraspinal CSF volumes across spontaneous intracranial hypotension stages and to test the clinical applicability of these measures.
\end{abstract}

MATERIALS AND METHODS: A cohort of 23 patients with spontaneous intracranial hypotension and 32 healthy controls was subjected to brain MR imaging and MR myelography with 1.5T imaging. An automatic threshold-based segmentation method was used to calculate intraspinal CSF volumes at initial hospitalization (spontaneous intracranial hypotension-initial), partial improvement (spontaneous intracranial hypotension-intermediate), and complete recovery (spontaneous intracranial hypotension-recovery) stages.

RESULTS: The mean intraspinal CSF volumes observed were the following: $95.31 \mathrm{~mL}$ for healthy controls, $72.31 \mathrm{~mL}$ for spontaneous intracranial hypotension-initial, $81.15 \mathrm{~mL}$ for spontaneous intracranial hypotension-intermediate, and $93.74 \mathrm{~mL}$ for spontaneous intracranial hypotension-recovery. Increased intraspinal CSF volumes were related to disease recovery $(P<.001)$. The intraspinal CSF volumes of patients before complete recovery were significantly lower than those of healthy controls. With the estimated intradural CSF volumes as a reference, the intraspinal CSF volume percentage was lower in patients with spontaneous intracranial hypotension with venous engorgement than in those without it $(P=.058)$.

CONCLUSIONS: With a threshold-based segmentation method, we found that spinal CSF hypovolemia is fundamentally related to spontaneous intracranial hypotension. Intraspinal CSF volumes could be a sensitive parameter for the evaluation of treatment response and follow-up monitoring in patients with spontaneous intracranial hypotension.

ABBREVIATIONS: $\mathrm{BH}=$ body height; $\mathrm{BMI}=$ body mass index; $\mathrm{BW}=$ body weight; 3D-SPACE = 3D sampling perfection with application-optimized contrasts by using different flip angle evolution; ECSF = estimated intradural CSF volume; DPE = diffuse pachymeningeal enhancement; $\mathrm{HC}=$ healthy control; MRM = MR myelography; $\mathrm{SIH}=$ spontaneous intracranial hypotension

$S^{\mathrm{p}}$ pontaneous intracranial hypotension $(\mathrm{SIH})$ has been diagnosed increasingly as an important cause of headaches. Orthostatic headache is the characteristic symptom of SIH caused by CSF leakage in the spine. Despite the term "SIH," CSF hypovole-

Received September 23, 2016; accepted after revision December 29. From the Departments of Radiology (H.-C.C., C.-H.C., C.C.-C.C., J.-W.C.) and Neurology (P.-L.C.), Taichung Veterans General Hospital, Taichung, Taiwan; Department of Medicine (H.-C.C.), National Yang-Ming University, Taipei, Taiwan; and College of Medicine (Y.-H.T., J.-W.C.), China Medical University, Taichung, Taiwan.

Please address correspondence to Jyh-Wen Chai, MD PhD, Department of Radiology, Taichung Veterans General Hospital, Taichung 407, Taiwan, 1650 Taiwan Blvd, Section 4, Taichung, Taiwan 407; e-mail: hubt@vghtc.gov.tw

三 Indicates article with supplemental on-line table.

http://dx.doi.org/10.3174/ajnr.A5134 mia may be the core of the condition. ${ }^{1}$ About $18 \%$ of patients with symptoms typical of SIH have normal CSF opening pressure. ${ }^{2,3}$ The diagnosis of SIH depends on various imaging characteristics and clinical symptoms. The commonly reported findings indicating CSF leakage are epidural fluid accumulation with a dilated epidural venous plexus, a triangular-shaped expansion of the neural sleeve, and an irregular linear signal lateral to the neural sleeve on spinal MR imaging or MR myelography (MRM)., Conventional brain MR imaging has demonstrated diffuse pachymeningeal enhancement (DPE), venous engorgement, and pituitary hyperemia in patients with $\mathrm{SIH} .{ }^{4}$ However, DPE and other brain MR imaging abnormalities are not observed in all patients with $\mathrm{SIH}$, especially in early-stage $\mathrm{SIH}{ }^{6}$

As the amount of CSF decreases, the buoyant force from in- 
tracranial CSF is reduced and the brain descends while patients are standing. This results in stretching of pain-sensitive structures on the brain surface and causes orthostatic headaches. However, CSF hypovolemia has only been linked indirectly as a causative factor of SIH in the literature. ${ }^{1}$ Researchers have used imaging signs to determine the severity of CSF hypovolemia, such as the number of vertebral bodies over which leaked CSF had spread in spine MR imaging and DPE in brain MR imaging. ${ }^{1}$ Therefore, in addition to morphologic alterations in the brain and spine, we hypothesized that quantifying intraspinal CSF volumes would be crucial to evaluating the hypovolemic status of patients with SIH. This important issue of direct monitoring of CSF volume depletion has not been discussed in the SIH literature, to our knowledge.

The aim of this study was to apply an automatic segmentation method to measure the entire CSF volume in the spine and detect CSF hypovolemia in patients with SIH. Furthermore, we compared intraspinal CSF volumes across SIH stages and tested the clinical applicability of these measures.

\section{MATERIALS AND METHODS}

\section{Subjects}

Between January 2012 and February 2016, 23 patients diagnosed with SIH (according to the criteria of Schievink et $\mathrm{al}^{7}$ or the "International Classification of Headache Disorders," $3 \mathrm{rd} \mathrm{ed}^{8}$ ) and treated in the neurology department at our hospital (Taichung Veterans General Hospital, Taichung, Taiwan) were enrolled in our study. During their hospital stays, patients received conservative treatment and a targeted epidural blood patch. Whole-spine MRM and conventional brain MR imaging were performed on patients simultaneously at 3 different time points. All patients underwent MR imaging on presentation/enrollment that was defined as SIH-initial. Some patients received a second MR imaging for an additional epidural blood patch when their clinical symptoms improved but did not resolve completely. These measurements are the SIH-intermediate. The mean time of MR imaging for SIH-intermediate measurement was $8.41 \pm 4.34$ days. If complete recovery was achieved following treatment, patients received another MR imaging, the SIH-recovery measurement. Complete recovery was defined as patients having no more headaches or other associated clinical orthostatic symptoms and no CSF leakage or other abnormalities detected in MRM or MR imaging. The mean time of MR imaging for SIH-recovery was $104.67 \pm 94.00$ days.

Thirty-two healthy volunteers with no known neurologic disorders, spine malformations, or previous spine operations were recruited as healthy controls (HCs). Whole-spine MRM was performed for each subject, and the only pathologic finding acceptable for inclusion was minor degenerative changes of the spine. Before enrollment, written informed consent was obtained from all subjects. This study was approved by the institutional review board of Taichung Veterans General Hospital.

\section{MR Imaging Acquisition}

Subjects underwent MRM and brain MR imaging on a 1.5T MR imaging scanner (Magnetom Aera; Siemens, Erlangen, Germany). Whole-spine MRM was performed with a 3D sampling perfection with application-optimized contrasts by using different flip angle evolution (3D-SPACE) sequence (Siemens). The MR imaging parameters were as follows: $\mathrm{TR}=3000 \mathrm{~ms}, \mathrm{TE}=560$ $\mathrm{ms}$, isotropic voxel size $=0.9 \mathrm{~mm}^{3}$, matrix size $=320 \times 320$ pixels, and $\mathrm{FOV}=200 \mathrm{~mm}$. Fat suppression and a generalized autocalibrating partially parallel acquisition imaging reconstruction with an acceleration factor of 2 were used. Images were acquired volumetrically in the coronal plane of the cervical-to-thoracic and thoracic-to-lumbar regions of the spine, parallel to the spinal curve with some overlapping. The acquisition time was 7 minutes 58 seconds for each segment. Conventional brain MR imaging included axial spin-echo T1-weighted images (TR/TE, $500 / 10 \mathrm{~ms}$ ) (2 minutes 12 seconds); axial fast-spin-echo T2weighted images (TR/TE, 3200/115 ms) (2 minutes 39 seconds); and gadolinium-enhanced spin-echo T1WI in the axial (2 minutes 25 seconds), sagittal ( 2 minutes 10 seconds), and coronal planes ( 1 minute 56 seconds). The contrast-enhanced study was performed by using $1.0 \mathrm{~mol} / \mathrm{L}$ of gadolinium chelate (gadobutrol, Gadovist 1.0; Bayer Schering Pharma, Berlin, Germany) with 0.1 mmol per kilogram of body weight.

\section{Intraspinal CSF Volume Measurement and Data Segmentation}

Before segmentation, a preprocessing of bias field correction was performed to reduce intensity inhomogeneity in 2 sets of 3DSPACE whole-spine MRM studies. The threshold-based algorithm of relative entropy has been proposed for use in clinical applications to enable reproducible and facile image segmentation. $^{9-12}$ With the advantages of selecting an optimal threshold value with local neighborhood information, the method could extract hyperintense CSF voxels from other hypointense background tissue automatically and robustly in 3D-SPACE wholespine MRM. After processing, we calculated CSF volumes by identifying all CSF-specific voxels from the tip of the odontoid process to the end of the dural sac. The volumes obtained were defined as intraspinal CSF volumes, including intra- and extradural CSF in the spinal canal at SIH-initial and SIH-intermediate measurements. For SIH-recovery measurements and HCs, intraspinal CSF volumes would be equal to intradural CSF because no CSF leakage occurred. Intraspinal CSF volumes were evaluated by using absolute data. We also estimated each subject's intradural CSF volumes by correlated parameters. The intraspinal CSF volume percentage was defined as the following: Intraspinal CSF Volumes of SIH-Initial / Estimated Intradural CSF Volumes.

\section{Brain Imaging Measurements}

Some qualitative indications in brain images were evaluated, including the following: pituitary volumes (in cubic centimeters) defined as the half-value of the product of the gland height, length, and width; venous engorgement, which appears as a convex upper surface of the sinus confluence on a sagittal view of postcontrast T1WI; and any DPE, defined as continuously enhanced dural matter.

\section{Statistical Analyses}

All data were analyzed in SPSS software (Version 18; IBM, Armonk, New York). Demographic data, intraspinal CSF volumes, 
Table 1: Demographic data

\begin{tabular}{cccc}
\hline Parameter & Patients with SIH & HCs & $P$ Value \\
\hline Age, y (mean) & $38.83 \pm 11.83$ & $35.56 \pm 10.02$ & .393 \\
Range & $23-58$ & $20-59$ & \\
BMI, kg/m (mean) $^{\mathrm{a}}$ & $24.97 \pm 5.80$ & $22.07 \pm 4.24$ & .078 \\
$\mathrm{BH}, \mathrm{m}($ mean) & $1.67 \pm 0.098$ & $1.67 \pm 0.078$ & .942 \\
$\mathrm{BW}, \mathrm{kg}($ mean) & $72.22 \pm 20.72$ & $61.48 \pm 12.79$ & .077 \\
Sex & & & .598 \\
Male (No.) & 8 & 10 & \\
Female (No.) & 15 & 22 & \\
\hline
\end{tabular}

a Mann-Whitney $U$ test.

${ }^{\mathrm{b}}$ Two-sample $t$ test.

and brain MR imaging findings were compared between HCs and the SIH-initial, SIH-intermediate, and SIH-recovery measurements of patients. We used Kolmogorov-Smirnov tests to verify normal distribution of continuous variables. The age, body mass index (BMI), body height (BH), body weight (BW), and intraspinal CSF volume data for subjects with SIH and HCs were not normally distributed and were therefore analyzed with nonparametric Mann-Whitney tests. The sexes between patients with $\mathrm{SIH}$ and HCs were analyzed by using a 2-sample $t$ test. The difference among SIH-initial, SIH-intermediate, and SIH-recovery measurements was analyzed with the paired $t$ test. The relationships between intraspinal CSF volumes of the HCs and SIH-recovery measurements with age, BMI, BH, BW, and sex were subjected to the Spearman rank-based correlation coefficient. For estimated intradural CSF volumes (ECSF), we used linear regression to fit parameters that correlated with the intraspinal CSF volumes in SIH-recovery measurements of patients and HCs. All tests were 2-tailed with statistical significance at $P<.05$.

\section{RESULTS}

Patient demographics and baseline characteristics are summarized in Table 1. There were no statistical differences in age, sex, BMI, BH, or BW between the patients with SIH and HCs. Most of our patients had a significant amount CSF leakage at the spinal nerve roots with epidural fluid accumulation. The extent of CSF leakage for each patient is summarized in the On-line Table. All patients received at least 1 targeted epidural blood patch without any complications. Five patients did not have follow-up MR imaging at symptoms partially improved. Five patients were found to not have achieved complete recovery. Comparisons of spinal and brain MR imaging data between HCs and patients with SIH are reported in Table 2.

\section{Intraspinal CSF Volumes}

The mean intraspinal CSF volumes obtained for the SIH-initial $(72.31 \mathrm{~mL})$ and SIH-intermediate $(81.15 \mathrm{~mL})$ stages differed significantly from those of the HCs $(95.31 \mathrm{~mL} ; P<.001$ and $P<.05$, respectively). However, the mean intraspinal CSF volumes obtained for the SIH-recovery stage $(93.74 \mathrm{~mL})$ were similar to those of the HCs $(P=.731)$. Intraspinal CSF volumes increased stepwise $(P<.001)$ in patients with SIH from SIH-initial to SIHintermediate to SIH-recovery.

\section{Estimated Intradural CSF Volumes}

Intraspinal CSF volumes of HCs and SIH-recovery measurements correlated moderately well and significantly with $\mathrm{BH}$ (correlation
Table 2: Spinal MR imaging parameters and brain MRI findings from HCs and patients with SIH at initial, intermediate, and recovery stages

\begin{tabular}{|c|c|c|c|}
\hline & & $\begin{array}{c}\text { Intraspinal } \\
\text { CSF Volumes } \\
(\mathrm{mL})\end{array}$ & $\begin{array}{c}\text { Pituitary } \\
\text { Volumes } \\
\left(\mathrm{cm}^{3}\right)\end{array}$ \\
\hline $\mathrm{HCs}(n=32)$ & & $95.31 \pm 15.48$ & \\
\hline \multirow[t]{3}{*}{$\mathrm{SIH}$} & $\operatorname{lni}(n=23)$ & $72.31 \pm 19.35$ & $0.60 \pm 0.20$ \\
\hline & $\operatorname{lnt}(n=18)$ & $81.15 \pm 25.41$ & $0.47 \pm 0.15$ \\
\hline & $\operatorname{Rec}(n=18)$ & $93.74 \pm 20.33$ & $0.36 \pm 0.14$ \\
\hline \multirow[t]{3}{*}{$P$ value ${ }^{a}$} & HCs vs $\mathrm{SIH}_{\mathrm{Ini}}$ & $<.001^{\mathrm{b}}$ & \\
\hline & HCs vs SIH Int & $<.05^{b}$ & \\
\hline & HCs vs $\mathrm{SIH}_{\mathrm{Rec}}$ & .731 & \\
\hline \multirow[t]{3}{*}{$P$ value ${ }^{c}$} & $\mathrm{SIH}_{\text {Ini }}$ vs SIH Int & $<.001^{b}$ & $<.001^{b}$ \\
\hline & $\mathrm{SIH}_{\text {Ini }}$ vs SIH $\mathrm{Sec}_{\mathrm{Re}}$ & $<.001^{b}$ & $<.001^{b}$ \\
\hline & $\mathrm{SIH}_{\text {Int }}$ vs SIH & $<.001^{\mathrm{b}}$ & .155 \\
\hline
\end{tabular}

Note:-Ini indicates initial; Int, intermediate, Rec, recovery.

${ }^{a}$ Mann-Whitney $U$ test.

b Significant.

c Paired $t$ test.

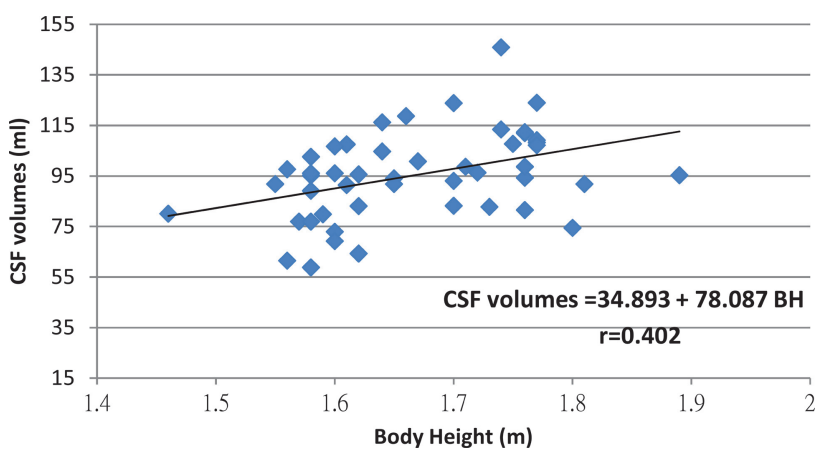

FIG 1. Linear regression equation and figure of intraspinal CSF volumes with $\mathrm{BH}$.

coefficient $=0.444, P=.002$ ), but not with BMI (correlation coefficient $=-0.033, P=.822)$, BW (correlation coefficient $=$ $0.073, P=.622)$, age (correlation coefficient $=-0.215, P=.134$ ), or sex (correlation coefficient $=-0.233, P=.164$ ). Because intradural CSF volumes varied across individuals, we estimated each subject's intradural CSF volumes by using a linear regression equation according to $\mathrm{BH}$ to see whether it could provide useful clinical information for diagnosis and treatment (Fig 1).

Relative to ECSF values, intraspinal CSF volumes in the SIHinitial $(P<.001)$ and SIH-intermediate $(P<.05)$ groups, but not the SIH-recovery group $(P=.772)$, were significantly reduced (Table 3).

\section{Diffuse Pachymeningeal Enhancement}

Among the 23 patients with SIH, 15 had DPE (Table 4). The mean intraspinal CSF volumes of patients with SIH and DPE $(70.14 \pm 18.74 \mathrm{~mL})$ did not differ significantly from those of patients with SIH without DPE $(76.36 \pm 21.10 \mathrm{~mL}, P=.388)$. The intraspinal CSF volume percentage (ie, quotient of the initial intraspinal CSF volumes and ECSF volumes) also did not differ significantly between patients with $\mathrm{SIH}$ with and without DPE $(P=.274)$.

\section{Venous Engorgement}

Among the 23 patients with SIH, 13 showed venous engorgement and the other 10 did not (Table 4). The intraspinal CSF volumes of 
patients with SIH with venous engorgement $(66.24 \pm 16.40 \mathrm{~mL})$ did not differ from those of patients without venous engorgement $(80.19 \pm 20.84 \mathrm{~mL}, P=.128)$. Although the intraspinal CSF volume percentage of patients with SIH with venous engorgement did not differ from that of those without venous engorgement $(P=.058)$, there seemed to be a trend toward patients with $\mathrm{SIH}$ with venous engorgement having a lower intraspinal CSF volume percentage.

\section{Pituitary Volumes}

As shown in Table 2, pituitary volumes were significantly larger in the SIH-initial than in the SIH-intermediate and SIHrecovery measurements (both $P<.001$ ). However, there was no significant difference in the SIH-intermediate and SIH-recovery pituitary volumes $(P=.155)$. Pituitary volumes did not differ significantly between patients with SIH with and without DPE $(P=.747$, Table 4$)$. However, the pituitary volumes of patients with $\mathrm{SIH}$ with venous engorgement were significantly greater than those of patients with $\mathrm{SIH}$ without venous engorgement $(P<.05$, Table 4$)$.

\section{Case Illustration}

MR imaging and MRM images obtained for a 57-year-old woman who was diagnosed with CSF leakage into the spine are shown in Fig 2. The initial brain MR imaging showed no evidence of DPE, venous engorgement, or pituitary hyperemia. The calculated intraspinal CSF volume for this patient was $68.36 \mathrm{~mL}$ in the initial stage. The estimated intradural CSF for this patient was $79.11 \mathrm{~mL}$, with an intraspinal CSF volume percentage of $86.5 \%$. The intradural CSF volume at complete recovery was $80.04 \mathrm{~mL}$.

MR imaging and MRM images obtained for a 34-year-old man who had orthostatic headache and was shown to have CSF leakage into the spine are shown in Fig 3. The calculated intraspinal CSF volume for this patient was $88.11 \mathrm{~mL}$ in the initial stage. DPE, venous engorgement, and pituitary hyperemia were noted in the patient's initial brain MR imaging. The estimated intradural CSF

Table 3: Comparison of intraspinal CSF volumes at each SIH stage relative to the ECSF value

\begin{tabular}{lcccc}
\hline & & \multicolumn{3}{c}{ SIH } \\
\cline { 3 - 5 } \multicolumn{1}{c}{ Parameter } & ECSF $(\boldsymbol{n}=23)$ & $\operatorname{Ini}(\boldsymbol{n}=23)$ & $\operatorname{Int}(\boldsymbol{n}=18)$ & $\operatorname{Rec}(\boldsymbol{n}=\mathbf{1 8})$ \\
\hline Intraspinal CSF volumes $(\mathrm{mL})$ & $93.03 \pm 13.23$ & $72.31 \pm 19.35$ & $81.15 \pm 25.41$ & $93.74 \pm 20.33$ \\
$P$ values, ECSF vs SIH & - & $<.001^{\mathrm{b}}$ & $<.05^{\mathrm{b}}$ & .772 \\
\hline
\end{tabular}

Note:-Ini indicates initial; Int, intermediate, Rec, recovery.

${ }^{\text {a }}$ Paired $t$ test.

bignificant.

Table 4: Comparison of MR imaging parameters between patients with SIH versus those without DPE and venous engorgement

\begin{tabular}{|c|c|c|c|c|c|c|}
\hline & \multicolumn{6}{|c|}{ Volume (Mean) } \\
\hline & $\begin{array}{l}\text { SIH with Ini } \\
\text { DPE }(n=15)\end{array}$ & $\begin{array}{l}\text { SIH without Ini } \\
\text { DPE }(n=8)\end{array}$ & $P$ Value $^{\mathrm{a}}$ & $\begin{array}{l}\text { SIH with Ini } \\
\text { Venous } \\
\text { Engorgement } \\
(n=13)\end{array}$ & $\begin{array}{l}\text { SIH without Ini } \\
\text { Venous } \\
\text { Engorgement } \\
(n=10)\end{array}$ & $P$ Value \\
\hline \multicolumn{7}{|l|}{ Spinal } \\
\hline Intraspinal CSF volumes (mL) & $70.14 \pm 18.74$ & $76.36 \pm 21.10$ & .388 & $66.24 \pm 16.40$ & $80.19 \pm 20.84$ & .128 \\
\hline Intraspinal CSF volume percentage & $78.59 \pm 29.27$ & $81.75 \pm 19.64$ & .274 & $70.87 \pm 17.74$ & $91.16 \pm 30.99$ & .058 \\
\hline \multicolumn{7}{|l|}{ Brain } \\
\hline Pituitary volumes $\left(\mathrm{cm}^{3}\right)$ & $0.61 \pm 0.20$ & $0.58 \pm 0.19$ & .747 & $0.67 \pm 0.15$ & $0.51 \pm 0.22$ & $<.05^{\mathrm{b}}$ \\
\hline
\end{tabular}

Note:-Ini indicates initial; Int, intermediate, Rec, recovery.

${ }^{a}$ Mann-Whitney $U$ test.

b Significant. for this patient was $103.32 \mathrm{~mL}$, with an intraspinal CSF volume percentage of $85.3 \%$. The intradural CSF volume at complete recovery was $109.15 \mathrm{~mL}$.

\section{DISCUSSION}

In this study, we obtained direct measurements of intraspinal CSF volumes in patients with $\mathrm{SIH}$ at 3 clinical stages and compared them with those of HCs. Although the intraspinal CSF volumes in the SIH-initial and SIH-intermediate measurements included leaked CSF in the extradural space and intradural CSF, stepwise increases in intraspinal CSF volumes were observed during the disease recovery. Furthermore, the mean intraspinal CSF volume was significantly lower in the SIH-initial and -intermediate disease stages than in the SIH-recovery stage or in HCs. These findings support the hypothesis that CSF hypovolemia is a core feature of SIH syndrome.

The normal intraspinal CSF volume range for healthy subjects has not been well-documented. In subjects without CSF leakage, intraspinal CSF volumes should be equal to intradural CSF volumes. Hogan et $\mathrm{al}^{13}$ reported 2 healthy volunteers with intradural CSF volumes of the spine plus nerve roots of 95 and $120 \mathrm{~mL}$. The only large-scale report was a study of 22 healthy volunteers having a mean intradural CSF volume of $81 \pm 13 \mathrm{~mL}$ (range, 52-103 $\mathrm{mL}) .{ }^{14}$ In that study, the original images were obtained with $3 \mathrm{D}$ balanced turbo-field echo pulse sequences and the regions for measurement were drawn on the images manually. For CSF volume calculation, the original images were transformed to an axial image of $1.9-\mathrm{mm}$ thickness with a $0.7-\mathrm{mm}$ spacing covering the whole spine. Manually drawing the outline of the spinal cord, spinal canal, and nerve root on every 10th axial image was performed. The intradural CSF volume of the spine was calculated as the following: Area of Spinal Canal - Nerve Roots and Spinal Cord $\times$ Interslice Distance.

Here, we used automatic threshold-based segmentation methods for CSF analysis to estimate the mean intradural CSF volumes of 32 HCs. Slightly larger intradural CSF volumes were observed in our study. The reason for the volume differences might be from the imaging sequences and analytic methods. In our experiment, a heavily $\mathrm{T} 2$-weighted $3 \mathrm{D}$ SPACE sequence was used to acquire whole spinal MRM, which provided higher isotropic spatial resolution and tissue contrast of CSF in whole spinal 


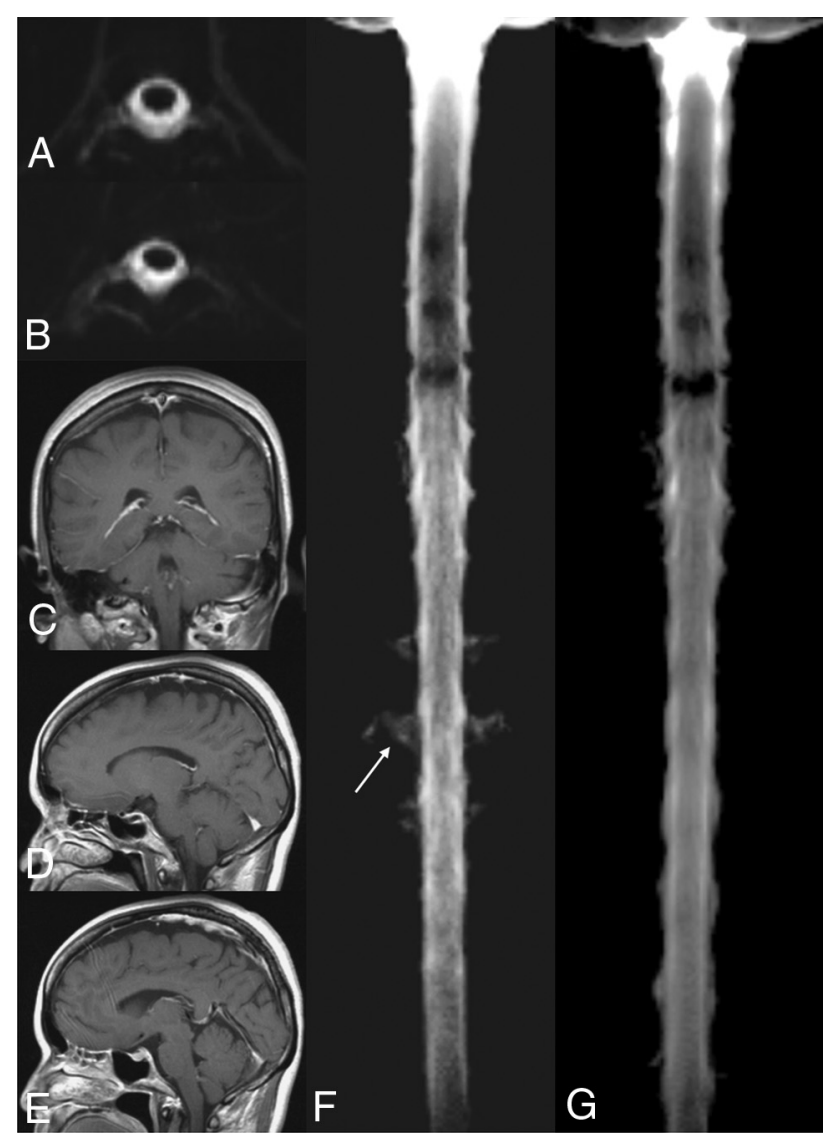

FIG 2. A 57-year-old woman diagnosed with CSF leakage in the spine with epidural fluid accumulation $(A)$ and CSF signals along the neural sleeve $(B)$ at the patient's initial MRM. The patient's initial brain MR imaging showed no evidence of DPE $(C)$, venous engorgement $(D)$, or pituitary hyperemia (E). 3D maximum intensity projection of the initial MRM (F) revealed CSF leakage along the neural sleeves at the T-spine (arrow) and reduced CSF volumes with lower CSF intensities of the dural sac compared with 3D MIP of her recovery MRM $(G)$.

images. ${ }^{15}$ We believed that sequence would be more sensitive for detecting the CSF voxels than using a balanced turbo-field echo sequence because the SPACE sequence has less susceptibility artifact-related image blurring and better contrast-to-noise ratio. ${ }^{16,17}$ Additionally, the automatic threshold-based segmentation method could effectively eliminate the discrepancy from intraand interoperator variability and increase the reliability in clinical applicability. Our cohort was younger (range, 39-59 years) than those in prior studies. For example, in Edsbagge et al, ${ }^{14}$ all participants were older than 64 years of age. Degenerative spinal changes, such as spurs or stenosis, might reduce intradural CSF volumes. However, we did observe a wide variation of intradural CSF volumes across individuals.

Using ECSF volumes as a reference to predict individual intraspinal CSF volumes, we discovered that patients with SIH had significantly lower intraspinal CSF volumes at their initial and intermediate measurements. No significant differences in intraspinal CSF volumes were found between the recovery measurements and the ECSF values. We presented 2 cases of SIH with about 20-mL differences in their intraspinal CSF volumes (68.36 versus $88.11 \mathrm{~mL}$ ) at the initial stage (Figs 2 and 3). It was difficult to determine whether their intraspinal CSF volumes were reduced

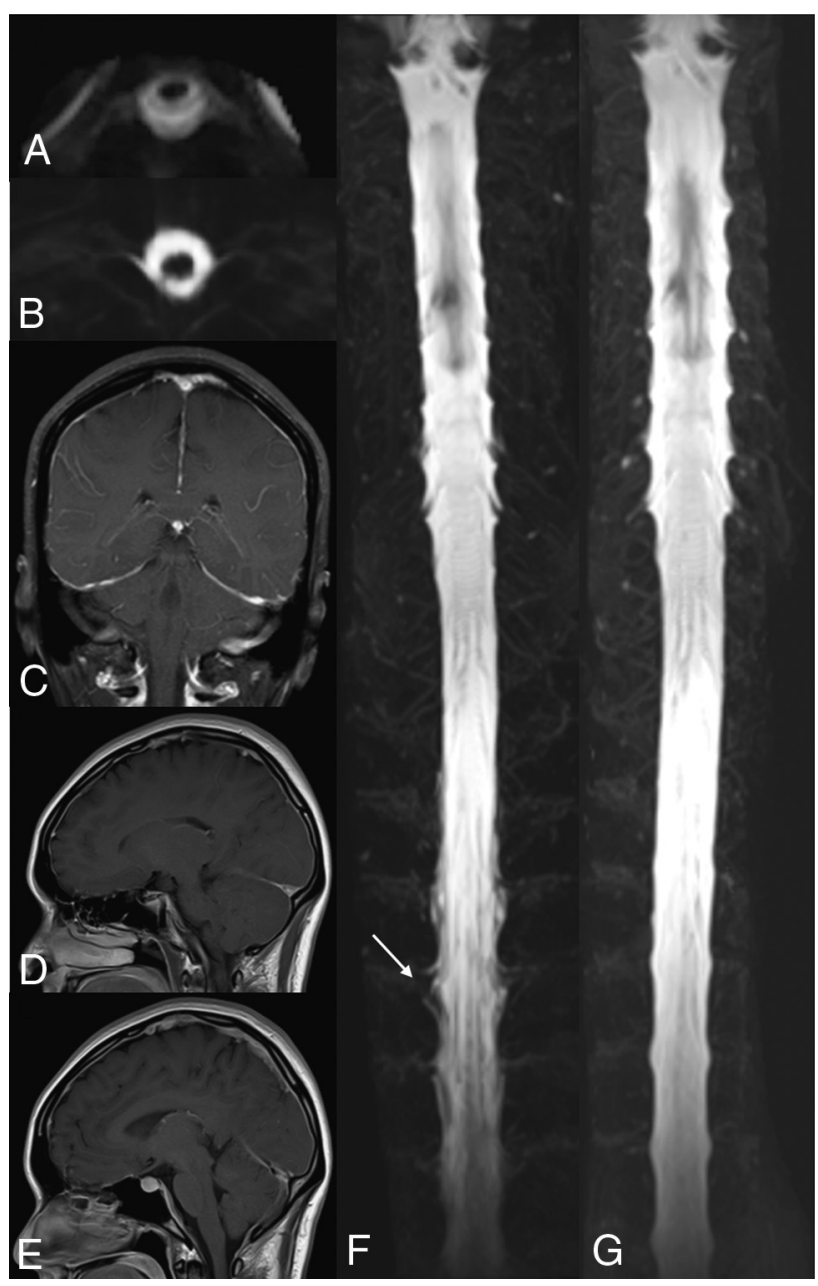

FIG 3. A 34-year-old man with orthostatic headache. CSF leakage at the spine with epidural fluid accumulation $(A)$ and CSF signals along the neural sleeve $(B)$ were seen at the patient's initial MRM. DPE $(C)$, venous engorgement $(D)$, and pituitary hyperemia $(E)$ were noted at the initial brain MR imaging. 3D maximum intensity projection of the initial MRM (F) revealed an irregular contour along the neural sleeves at the T-spine, indicating CSF leakage (arrow) and reduced CSF volumes with lower CSF intensities of the dural sac compared with the 3D MIP of his recovery MRM $(G)$.

by only using the absolute volumes. The male patient had higher ECSF than the female patient (103.32 versus $79.11 \mathrm{~mL}$ ). With ECSF as a reference, both patients had significantly reduced CSF volumes with the intraspinal CSF percentage of about $85 \%$ causing orthostatic headache and other image abnormalities. Their intradural CSF volumes during recovery were 80.04 and 109.15 $\mathrm{mL}$, respectively. Therefore, ECSF volumes might serve as an important reference for the diagnosis and evaluation of disease severity.

SIH-associated brain changes can be explained by the MonroKellie doctrine, ${ }^{18}$ wherein decreases in intracranial CSF volumes are accompanied by increased volumes of brain tissue (eg, pituitary hyperemia) or vascular structures (DPE or venous engorgement). In our study, intraspinal CSF volumes and volume percentage in patients with SIH with DPE $(n=15)$ were similar to the values obtained for patients without DPE $(n=8)$. However, the potential effect of venous engorgement on these parameters was less clear. Although there was no significant difference of intraspi- 
nal CSF volumes between patients with SIH with $(n=13)$ and without $(n=10)$ venous engorgement, there was a strong trend toward a difference in intraspinal CSF volume percentage between these 2 groups (borderline $P$ value $=.058$ ). This trend may become a robustly significant difference with a larger sample size. Venous engorgement was present in patients with more severe CSF depletion and might not be present in those with mild CSF volume loss. In a previous study, we also demonstrated that DPE or venous engorgement reflected a more severe disease status and may not be seen in patients with mild disease. ${ }^{6}$ Therefore, we believe that intraspinal CSF volumes and, especially, intraspinal CSF volume percentage may be sensitive markers of SIH progression. Spine MR imaging has also been reported useful for diagnosing SIH, especially at early stages. ${ }^{19}$

Pituitary hyperemia has also been thought to be a sensitive imaging marker of SIH. All the patients in this study had an enlarged pituitary gland, and the pituitary volumes decreased in relation to symptomatic improvement. Pituitary volumes were significantly greater in patients with venous engorgement than in those without it. Although pituitary volumes may reflect disease severity, pituitary volumes vary greatly across individuals, with larger volumes often being seen in young women. ${ }^{20}$ The maximum height was seen in the women between 20 and 40 years of age and declined with age. ${ }^{21}$ Twelve female patients achieved complete recovery in our study. Their pituitary volumes did not differ from those of male patients. Six of them are between 20 and 40 years of age, and the other 6 are older than 40 years of age. The height and pituitary volume were not significantly different between these 2 groups. Two patients younger than 40 years of age and 1 patient older than 40 years of age had convex-shape pituitary glands. Even though SIH usually occurred in young women, the pituitary size was still variable among subjects. Therefore, using absolute pituitary volumes as a diagnostic criterion of $\mathrm{SIH}$ is inappropriate. However, relative change in pituitary volumes is a good parameter for evaluation of treatment effects.

In this study, we found that intradural spinal CSF volumes correlated positively with $\mathrm{BH}$. No correlations were observed with BMI, BW, age, and sex. In previous reports, no correlation was found between intradural $\mathrm{CSF}$ and $\mathrm{BH},{ }^{14}$ and inverse correlations of lumbosacral intradural CSF with BMI have been reported. ${ }^{13,22}$ The number of vertebrae has been used as an indicator of intradural CSF volume. ${ }^{1}$ Therefore, we thought that lower limb length might explain the variance in the relationship between $\mathrm{BH}$ and intradural CSF volumes across individuals. Additional large-scale studies may better uncover the relationships among clinical findings, brain imaging findings, and intracranial and intraspinal CSF volumes.

Our study has some limitations. First, because of the difficulty of the autosegmentation method in separating extradural CSF from intradural CSF in current status, the intraspinal CSF measurements included both extra- and intradural spaces in SIHinitial and SIH-intermediate measurements. Hence, the CSF that leaked through dural defects was also included in our calculations using our segmentation method. Even though the intraspinal CSF volumes in SIH-initial and SIH-intermediate measurements were still significantly lower than those of healthy controls and SIHrecovery measurements, most of our patients had a significant amount CSF leakage at the spinal nerve roots with epidural fluid accumulation. Therefore, if we took account of only the intradural CSF, we believe that the CSF volumes would be much lower than those in healthy controls in the patients with SIH. Further study with methods that can differentiate intradural CSF from extradural CSF might elucidate the dynamic changes in patients with SIH.

Second, our sample size was small, and intracranial CSF volumes were not measured. Studies with larger sample sizes with measurements of both intracranial and intraspinal CSF volumes may provide more information. Third, although hypovolemia is fundamentally related to $\mathrm{SIH}$, individual variations of intradural CSF volumes do exist. The intraspinal CSF volumes could help monitor treatment effects as well. On the other hand, the method by which intradural CSF volumes are estimated for subjects will be important in making clinical application and accurate diagnoses feasible. ECSF volumes determined on the basis of $\mathrm{BH}$ as in our study appear to reflect clinical status well and could be used to help determine whether further treatment is needed. However, the correlation between intradural CSF volumes and $\mathrm{BH}$ was not high enough. It still needs to be integrated with clinical symptoms and other imaging findings. How to estimate intradural CSF volumes better still needs to be investigated by using other parameters and larger sample sizes.

\section{CONCLUSIONS}

With a threshold-based segmentation method, we demonstrated that spinal CSF hypovolemia was fundamentally related to SIH and may serve as an early detection method before abnormalities are detectable by conventional brain MR imaging. Intraspinal CSF volumes can be used as a sensitive parameter in treatmentresponse monitoring and in SIH follow-up evaluations.

\section{REFERENCES}

1. Miyazawa K, Shiga Y, Hasegawa T, et al. CSF hypovolemia vs intracranial hypotension in "spontaneous intracranial hypotension syndrome." Neurology 2003;60:941-47 Medline

2. Mokri B, Hunter SF, Atkinson JL, et al. Orthostatic headaches caused by CSF leak but with normal CSF pressures. Neurology 1998; 51:786-90 CrossRef Medline

3. Spero M, Lazibat I, Stojic M, et al. Normal pressure form of the spontaneous intracranial hypotension: a case report with pituitary enlargement and asymptomatic pituitary haemorrhage. Neurol Sci 2011;32:933-35 CrossRef Medline

4. Mokri B. Spontaneous CSF leaks: low CSF volume syndromes. Neurol Clin 2014;32:397-42 CrossRef Medline

5. Yoo HM, Kim SJ, Choi CG, et al. Detection of CSF leaks in spinal CSF leak syndrome using MR myelography: correlation with radioisotope cisternography. AJNR Am J Neuroradiol 2008;29:649-54 CrossRef Medline

6. Tung H, Liao YC, Wu CC, et al. Usefulness of phase-contrast magnetic resonance imaging for diagnosis and treatment evaluation in patients with SIH. Cephalalgia 2014;34:584-93 CrossRef Medline

7. Schievink WI, Maya MM, Louy C, et al. Diagnostic criteria for spontaneous spinal CSF leaks and intracranial hypotension. AJNR AmJ Neuroradiol 2008;29:853-56 CrossRef Medline

8. Headache Classification Committee of the International Headache Society (IHS). The International Classification of Headache Disorders, 3rd edition (beta version). Cephalalgia 2013;33:629-808 CrossRef Medline 
9. Chang CI, Chen K, Wang J, et al. A relative entropy-based approach to image thresholding. Pattern Recognition 1994;27:1275-89 CrossRef

10. Zhang H, Fritts JE, Goldman SA. Image segmentation evaluation: a survey of unsupervised methods. Computer Vision and Image Understanding 2008;110:260-80 CrossRef

11. Chen X, Wang R, Cao Y, et al. A novel evaluation method based on entropy for image segmentation. Procedia Engineering 2012;29: 3959-65 CrossRef

12. Liang YC, Cuevas, JR. An automatic multilevel image thresholding using relative entropy and meta-heuristic algorithms. Entropy 2013; 15:2181-209 CrossRef

13. Hogan QH, Prost R, Kulier A, et al. Magnetic resonance imaging of cerebrospinal fluid volume and the influence of body habitus and abdominal pressure. Anesthesiology 1996;84:1341-49 Medline

14. Edsbagge M, Starck G, Zetterberg H, et al. Spinal cerebrospinal fluid volume in healthy elderly individuals. Clin Anat 2011;24:733-40 CrossRef Medline

15. Hodel J, Lebret A, Petit E, et al. Imaging of the entire cerebrospinal fluid volume with a multistation 3D SPACE MR sequence: feasibility study in patients with hydrocephalus. Euro Radiol 2013;23: 1450-58 CrossRef Medline

16. Kojima S, Suzuki K, Hirata M, et al. Depicting the semicircular ca- nals with inner-ear MRI: a comparison of the SPACE and TrueFISP sequences. J Magn Reson Imaging. 2013;37:652-59 CrossRef Medline

17. Ucar M, Tokgoz N, Damar, C., et al. Diagnostic performance of heavily $\mathrm{T} 2$-weighted techniques in obstructive hydrocephalus: comparison study of two different $3 \mathrm{D}$ heavily $\mathrm{T} 2$-weighted and conventional T2-weighted sequences. Jpn J Radiol 2015;33:94 CrossRef Medline

18. Mokri B. The Monro-Kellie hypothesis: applications in CSF volume depletion. Neurology 2001;56:1746-48 CrossRef Medline

19. Watanabe A, Horikoshi T, Uchida M, et al. Diagnostic value of spinal MR imaging in spontaneous intracranial hypotension syndrome. AJNR Am J Neuroradiol 2009;30:147-51 Medline

20. MacMaster FP, Keshavan M, Mirza, Y et al. Development and sexual dimorphism of the pituitary gland. Life Sci 2007;80:940-44 CrossRef Medline

21. Doraiswamy PM, Potts JM, Axelson DA, et al. MR assessment of pituitary gland morphology in healthy volunteers: age- and genderrelated differences. AJNR Am J Neuroradiol 1992;13:1295-99 Medline

22. Sullivan JT, Grouper S, Walker MT, et al. Lumbosacral cerebrospinal fluid volume in humans using three-dimensional magnetic resonance imaging. Anesth Analg 2006;103:1306-10 CrossRef Medline 